\title{
Wireless Network Virtualization by Leveraging Blockchain Technology and Machine Learning
}

\author{
Ashish Adhikari, Danda B. Rawat \\ Data Science and Cybersecurity Center (DSC ${ }^{2}$ ) \\ EECS Department, Howard University \\ Washington, DC 20059, USA \\ Email: db.rawat@ieee.org
}

\author{
Min Song \\ Dept. of Electrical \& Computer Engineering \\ Stevens Institute of Technology \\ Hoboken, NJ 07030, USA \\ E-mail: min.song@stevens.edu
}

\begin{abstract}
Wireless Virtualization (WiVi) is emerging as a new paradigm to provide high speed communications and meet Qualityof-Service (QoS) requirements of users while reducing the deployment cost of wireless infrastructure for future wireless networks. In WiVi, Wireless Infrastructure Providers (WIPs) sublease their RF channels through slicing to Mobile Virtual Network Operators (MVNOs) based on their Service Level Agreements (SLAs) and the MVNOs independently provide wireless services to their end users. This paper investigates the wireless network virtualization by leveraging both Blockchain technology and machine learning to optimally allocate wireless resources. To eliminate double spending ( $a k a$ over-committing) of WIPs' wireless resources such as RF channels, Blockchain - a distributed ledger technology is used where a reputation is used to penalize WIPs with past double spending habit. The proposed reputation based approach helps to minimize extra delay caused by double spending attempts and Blockchain operations. To optimally predict the QoS requirements of MVNOs for their users, linear regression - a machine learning approach - is used that helps to minimize the latency introduced due to (multiple wrong) negotiations for SLAs. The performance evaluation of the proposed approach is carried out by using numerical results obtained from simulations. Results have shown that the joint Blockchain and machine learning based approach outperforms the other approaches.
\end{abstract}

\section{INTRODUCTION}

Wireless Virtualization is an emerging technology to provide a feasible means to accommodate the QoS demands of end users by creating an efficient abstraction for sharing limited radio resources. In $\mathrm{WiVi}$, Wireless Infrastructure Providers (WIPs) sublease their RF channels through slicing to Mobile Virtual Network Operators (MVNOs) based on Service Level Agreements (SLAs). For service provisioning, the end users directly negotiate with the respective MVNOs. Note that most of the radio resources owned by WIPs remain idle

This work was supported in part by the US NSF grant CNS-1650831.

WiseML 2019, May 15-17, 2019, Miami, FL, USA

(C)2019 Association for Computing Machinery.

ACM ISBN 978-1-4503-6769-1/19/05 ...\$15.00

https://doi.org/10.1145/3324921.3328790 and are rarely operating at their maximum capacity $[1,2]$. Thus, wireless virtualization provides a promising solution to meet the increasing demands of wireless users while reducing the deployment cost of wireless infrastructure. A wireless virtualization model facilitates WIPs to share unused RF channels for enabling MVNOs to create virtual networks to serve end users.

In this paper, we leverage Blockchain technology and machine learning to optimally allocate wireless resources for wireless network virtualization where the Blockchain technology helps MVNOs sublease the RF slices from trustworthy WIPs (who do not double spend their resources) and the linear regression based machine learning approach helps MVNOs to predict the data rate requirements of users (which is used to negotiate with WIPs for RF slices). In Blockchain enabled wireless virtualization, we use a reputationbased approach which looks at recent past blocks in a Blockchain to find trustworthy WIPs and lets the WIPs to operate in real-time for allocating their slices to MVNOs. The main reason of using reputation-based approach is that we do not want to introduce extra delay because of Blockchain operations while allocating RF slices from WIPs to MVNOs. Furthermore, to optimally predict the QoS requirements of MVNOs for their users, linear regression - a machine learning approach - is used that helps to minimize the latency introduced due to (multiple wrong) negotiations for SLAs.

Recently, there have been several efforts to enhance the utilization of RF spectrum using different approaches such as dynamic spectrum access in cognitive radio networks $[1,3]$ and virtualization [2] to serve wireless users. Recent related works along our proposed work include [4-9]. Contract based resource allocation in wireless networks has been presented in [4] without considering the real-time negotiations for QoS requirements. Reinforcement learning based approach has been proposed in [5] to facilitate RF slicing for $5 \mathrm{G}$ networks which does not address the double spending of RF slices. Similarly, a dynamic price and frequency negotiation framework has been proposed in $[6,9]$ where the proposed approach adapts price of RF channels according to the number of MVNOs and requests from them. To prevent RF channel from over allocation by WIPs, a Blockchain based scheme has been presented in [7] to completely avoid double spending of wireless resources. This approach introduces an extra delay because of the Blockchain operations for processing each transaction to avoid any double spending in a 
real-time manner. In a real-time high traffic environment of mobile network, latency induced by such mechanisms can be a major cause for SLA violation and degradation in QoS. An anonymous reputation system has been proposed for vehicular ad-hoc networks (VANETs) in [8] without considering machine learning with Blockchain. Furthermore, renegotiation because of wrong QoS demands, poor channel quality, and system induced latencies may result in SLA violations in wireless virtualization. Currently, since there is no standard and widely accepted model to predict randomly changing user requests, the negotiations with WIPs are done by MVNOs as requests for data rates are received from end users. This introduces high delay in case of renegotiation or high interference in the network because of users who are operating without their QoS requirements being met. As none of the presented approaches consider a joint Blockchain technology and machine learning integration suitable for $\mathrm{WiVi}$ architecture, we propose a model to prevent WIPs from double spending RF slices while enabling MVNOs to predict QoS requirements in order to serve wireless users by updating their SLAs on the fly.

The rest of paper is organized as follows. Section 2 presents the system model comprising of the proposed integration of Blockchain technology and linear regression. Section 3 presents a formal analysis of the proposed approach for predicting the total data rate request and optimal $\mathrm{RF}$ slice selection. Performance evaluation is presented in Section 4. Finally, conclusions are presented in Section 5.

\section{SYSTEM MODEL}

In this paper, we consider a system model (as shown in Fig. 1) with $\mathcal{W}=\{1,2, \ldots, w, \ldots, W\}$ WIPs where each $w \in \mathcal{W}$ owns a set of RF slices represented as $\mathcal{Q}_{w}=\{1, \ldots \ldots Q\}$. These RF slices are subleased to MVNOs for a given time and location. The system model is considered to have $\mathcal{M}=$ $\{1,2, \ldots, m, \ldots, M\}$ MVNOs who serve their subscribed wireless users, as shown in Fig. 1.

\subsection{Blockchain Technology for WiVi}

In Blockchain enabled wireless network virtualization, $\mathrm{RF}$ channels are sliced into multiple slices to sublease to MVNOs. Note that the WIPs participating in wireless virtualization use public and private key combination as in Bitcoin systems [10]. The Blockchain managers of corresponding WIPs store service profiles on the Blockchain and verify WIPs' identities using public keys. Each Block, as shown in Fig. 2, in Blockchain for wireless virtualization consists of transactions representing bandwidth allocation information, maximum power allowed in the channel, supported data rates, reputation score, etc. The reputation score along with information in SLAs is used by the MVNOs while negotiating with WIPs and serving their end users.

\subsubsection{Preventing Double Spending by a WIP}

To completely avoid the double spending of the same RF slice by a given WIP, a given WIP subleases the RF slices to a MVNO where the given WIP is a source and the given MVNO is the recipient/destination as in [10]. The availability of RF slice is verified by a subgroup of participating WIPs and MVNOs that act as validators in the blockchain network. Similarly, when a given MVNO doesn't need the leased RF slices, it returns the RF slices to WIP where the given MVNO is a source and WIP is the recipient in form of

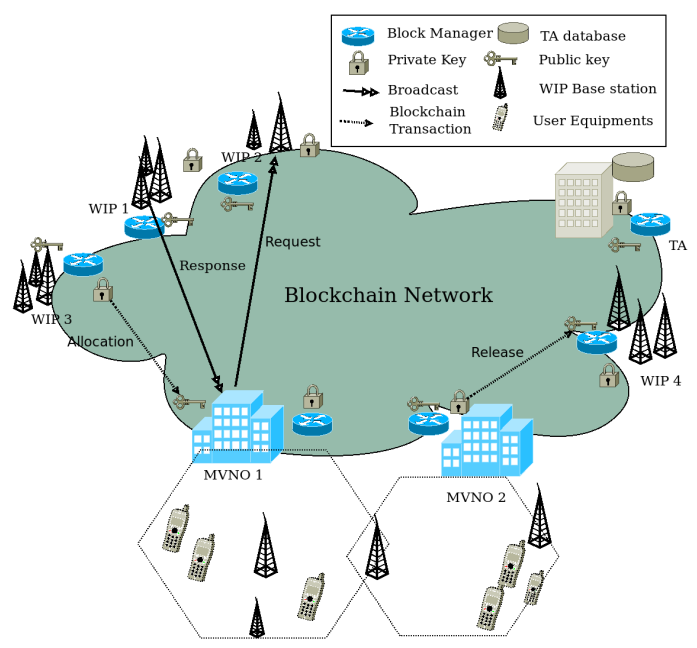

Figure 1: Wireless network virtualization model with Blockchain technology.

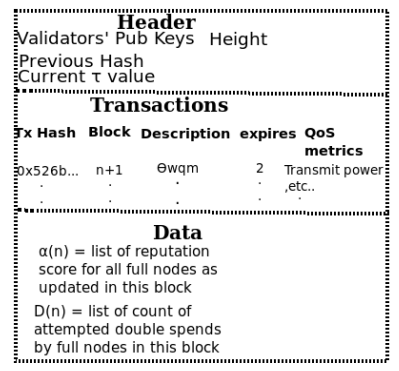

Figure 2: Proposed structure for blocks where data segment is used to keep extra data like reputation score and count of double spending attempts.

a blockchain transaction. However, when double-spending of same RF slice is checked at the time of allocation in wireless virtualization, it introduces extra delay because of the nature of the Blockchain operations in real-time where everyone participating has to sign the block.

Thus, to minimize delay caused by real-time double spending checking process in wireless virtualization, we introduce reputation score of WIPs by using its footprint of double spending in blocks (as discussed in sub-section 3.1). Note that when double spending of a RF slice happens from a WIP to multiple MVNOs, end-users served by those MVNOs will not be able to meet their QoS requirements when all MVNOs are fully loaded with users. We penalize the offending WIPs to minimize double spending through reputation score which is used by MVNOs while selecting the WIPs for subleasing RF slices.

\subsubsection{Penalizing the Double Spending WIPs}

The Trusted Authority (TA) such as the FCC in the US, as shown in Fig. 1, is responsible for allocating RF channels to WIPs by minting all tokens representing $\mathrm{RF}$ channels to be used in the Blockchain network as $\theta=\left\{\theta_{11}, \theta_{12} \ldots \theta_{w q}\right\}$. Note that there are two types of participants in the blockchain network: 1) WIPs who own primary infrastructure and RF slices, and are the actual owners of RF channels; and 2) MVNOs who do not own any physical infrastructure and RF resources but only temporarily sublease tokens (representing RF slices) from WIPs and return tokens back to respective WIP when not needed. In case of failure to return tokens or 
acting to violate the agreed terms, the SLA can be used to penalize the offending MVNOs. Furthermore, transactions are carried out between WIPs to borrow RF slices among WIPs for enabling dynamic load balancing among the WIPs in case of need as agreed upon in respective SLAs.

Transactions: Before any transaction is initiated, a request must be made by MVNO $m \in \mathcal{M}$ to WIP $w \in \mathcal{W}$ for a channel $\left(q_{w}\right)$ for an interval depending on its predicted total required data rate $\left(r^{\prime}\right)$ by using the Blockchain network. In return, WIP $w$ responds with acknowledgment through another broadcast and assignment of token $\theta_{w q}$ to the MVNO $m$. This allocation represents a transaction $\theta_{w q}^{m}$ and is verified by $m$ after it receives proof through confirmation in the blockchain. Then, $m$ serves its end users $(u)$ through the token allocated to it by $\theta_{w q}^{m}$ until the allocation expires after which the MVNO must conduct a transaction to return the token back to respective WIP or renew it.

For all transactions belonging to WIP $w$ i.e. $\left(\theta_{w}=\sum_{\forall q, m} \theta_{w q}^{m}\right)$ in block $B_{n+1}$, a validator is selected from a pre-specified set of validators to update the number of double spending attempts as detected by the validator. This is counted as $d_{w(n+1)}$ for the WIP $w$ when creating a new block $B_{n+1}$. This count is then used to update the reputation score for $w$ in the newly created block $B_{n+1}$ using the Algorithm 1 .

Reputation Scheme: Reputation update is regulated by validators that append new reputation scores for all WIPs with transactions into the new block as they add the block to the Blockchain. By using the data segment in blocks as illustrated in Fig. 2, we are able to maintain reputation scores in the Blockchain. Then, the reputation scores can be leveraged to discourage double spending among WIPs. Since a higher $\alpha_{w(n+1)}$ means that the WIP $w$ has the higher reputation and is expected to receive the most number of contracts from MVNOs which the WIP can choose from (Algorithm 3). Thus, the WIPs are motivated to minimize or avoid double spending of their RF slices for higher number of requests that could lead to their higher revenues. The reputation score update process is described in Algorithm 1.

\subsection{Linear Regression for Estimating Data Rate}

As a part of holistic framework for contract optimization through SLAs, MVNOs require as accurate as possible prediction of total data rate requirement to avoid undue system induced latencies. A machine learning based linear regression model is used for predicting total data rate requirement of a MVNO to meet QoS requirements of its users. Note that if a MVNO under-predicts the data rate requirement for its users, it has to renegotiate with WIPs and update their SLAs to meet the QoS requirements of its users. This introduces higher delay because of renegotiation as well as updating of SLAs, and higher interference in the systems from users who are running but not meeting their QoS requirements. Thus, the linear regression based data rate prediction model is used to predict total data rate requirement for users of MVNOs for the next cycle. The linear regression model is formally presented in Section 3.2.

Our goal in this paper is to combine Blockchain based reputation system and machine learning based prediction capabilities to investigate the enhancement on overall perfor-

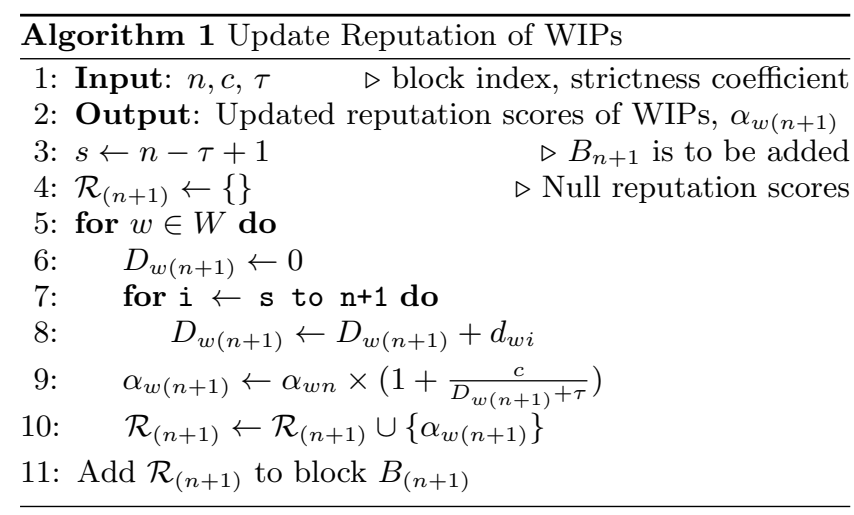

mance of MVNOs in WiVi scenario.

\section{PROPOSED APPROACH}

This section provides an analysis of the proposed approach.

\subsection{Reputation Score Calculation for WIPs}

In order to allow for a better optimization of incentive and punishment mechanism which can be managed either by collective voting of participating validators or system internal variables like growth rate of double spending in specified time, we use $\tau$ as the strictness coefficient to update the reputation score $\left(\alpha_{w(n+1)}\right)$ for WIP $w$ in the newly added block $B_{(n+1)}$. Formally, $\tau$ is defined as the number of blocks to traverse backwards starting at block $B_{n}$ in order to obtain the total double spending done by $w$ in its transactions for the blocks being examined. Calculated as shown in (1), $D_{w(n+1)}$ is used for updating the reputation score for WIP $w$ in the new block $B_{n+1}$.

$$
D_{w(n+1)}=\sum_{i=n-\tau+1}^{n+1} d_{w i},
$$

where $d_{w i}$ represents the number of double spending attempts by WIP $w$ in interval block $i$ was added to the blockchain. The updated reputation score $\left(\alpha_{w(n+1)}\right)$ for WIP $w$ is expressed as:

$$
\alpha_{w(n+1)}=\alpha_{w n} \times\left(1+\frac{c}{D_{w(n+1)}+\tau}\right),
$$

where $\alpha_{w n}$ is the reputation score in the previous block $B_{n}$ for a WIP $w$ and $c>0$ is a constant. The process of computing WIP's reputation score is expressed as Algorithm 1.

\subsection{Rate Prediction Using Linear Regression}

One of the popular machine learning models for predicting values, a linear regression model [11] is used to predict the total required data rate for maintaining user QoS for each MVNO. The linear regression model can be expressed for an interval $i$ (i.e., update interval) as

$$
r_{i}^{\prime}=\beta_{0}^{i-1}+\beta_{1}^{i-1} r_{i-1},
$$

where parameters $\beta_{0}$ and $\beta_{1}$ represent regression coefficients. $r_{i}^{\prime}$ represents the predicted total data rate requirement for MVNO for interval $i$, and $r_{i}$ represents the observed total data rate requirement for interval or cycle $i$. Here, $r_{i-1}$ is used as the training data rate for next cycle $i$. Prediction error $\left(\epsilon_{i}\right)$ for the cycle $i$ can be written as

$$
\epsilon_{i}=r_{i}-r_{i}^{\prime}
$$




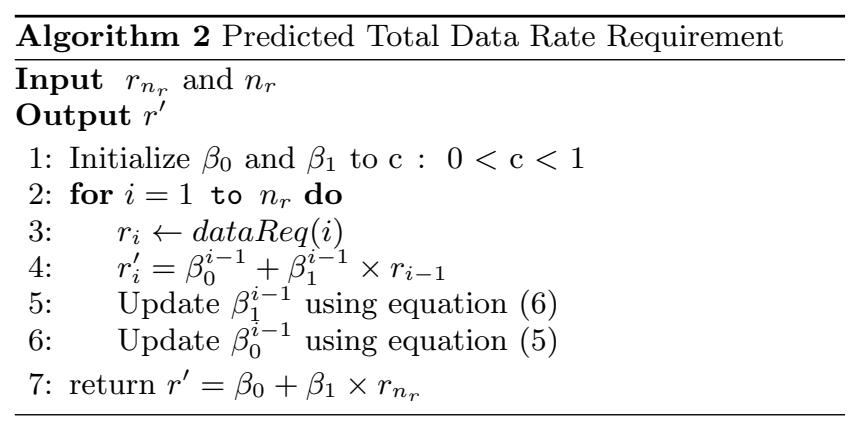

To incrementally adjust the regression coefficients [11, 12], $n_{r}$ data points representing the actual observations of data rate requirement for MVNO $m$ are used. The updated coefficients are then used to make a prediction for total data rate requirement which is then used to initiate $\mathrm{RF}$ channel negotiations to minimize system induced latency. The regression coefficients for interval $i$ can be updated incrementally using least squares method as

$$
\beta_{0}^{i}=\bar{r}_{i}-\beta_{1}^{i} \bar{r}_{i-1},
$$

where, $\bar{r}_{i}$ is the mean of all observed data rate upto interval $i$. Similarly, for $n_{r}$ data points, $\beta_{1}^{i}$ can be updated as

$$
\beta_{1}^{i}=\frac{\sum_{k=1}^{i}\left(r_{k-1}-\bar{r}_{k-1}\right)\left(r_{k}-\bar{r}_{k}\right)}{\sum_{k=1}^{i}\left(r_{k-1}-\bar{r}_{k-1}\right)^{2}} .
$$

The regression coefficients have to be iteratively adjusted with changing data points. The algorithmic steps are listed as Algorithm 2.

\subsection{Optimal RF Slice Selection for WiVi}

For a given RF channel $\left(q_{w}\right)$ with its bandwidth in $\mathrm{Hz}$ and signal-to-interference-plus-noise-ratio (SINR), the achievable data rate $r_{q_{w}}^{u}$ over the channel for a user $u$ of MVNO $m$ can be calculated in bits per second using Shannon capacity as

$$
r_{q_{w}}^{u}=\omega_{q_{w}}^{u} \times \log _{2}\left(1+S I N R_{q_{w}}^{u}\right),
$$

where $\omega_{q_{w}}^{u}$ represents the bandwidth of a the RF slice assigned to user $u$ of MVNO $m$ and $S I N R_{q_{w}}^{u}$ represents the SINR measured at $u$.

The SLA between a MVNO and a WIP includes a list of available RF channels $\left(Q_{w}\right)$ that can be subleased to MVNO $m$ from WIP $w$, the price $C_{q_{w}}$ for a channel $q \in Q_{w}$, geolocation of the WIP, reputation score of WIP, etc. Furthermore, by using the data rate requests and signal quality information obtained from MVNO $m$ 's end users overtime, and $m$ 's predicted total data rate requirement $\left(r^{\prime}\right)$, the MVNO $m$ can estimate and start negotiations for the optimal RF channels necessary to serve its end users even before its end user requests arrive. The optimal channel $q_{w}$ is the channel with highest utility value calculated using (8) and the Algorithm 3. The subleasing and release of the RF slices is synchronized through broadcast mechanisms and Blockchain transactions which is illustrated in Fig. 2. The utility value $T_{q_{w}}$ for each RF slice $q$ of WIP $w$ can be calculated as

$$
T_{q_{w}}=\frac{\hat{\alpha_{w}} \times r_{q_{w}}}{C_{q_{w}}}
$$

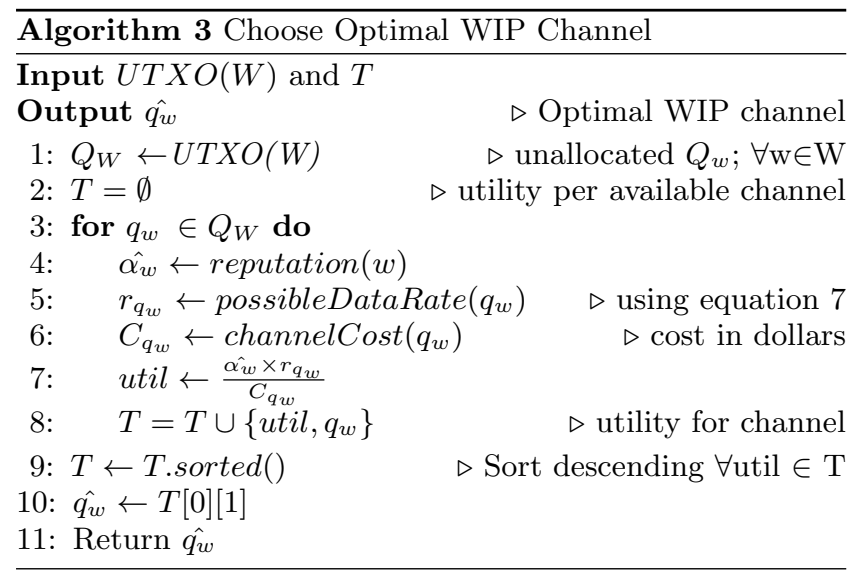

where $\hat{\alpha_{w}}$ represents the reputation score calculated for WIP $w, r_{q_{w}}=\sum_{\forall u} r_{q_{w}}^{u}$ represents the total data rate calculated for channel $q_{w}$ subleased by MVNO $m$ from WIP $w$, and $C_{q_{w}}$ is the price per $\mathrm{Hz}$ for channel. Specifically, a given MVNO $m$ can use Algorithm 3 to find the optimal channel from WIP $w$ until the required data rate is fulfilled as

$$
r^{\prime} \leq \sum_{q_{w} \in \hat{Q}_{w}} r_{q_{w}}
$$

where $r^{\prime}$ is the predicted total required data rate requirement and $\hat{Q}_{w}$ is the set of channels allocated to the MVNO $m$. The channel selection steps are summarized as Algorithm 3 where the given MVNO $m$ selects the best channel that meets total data rate requirements (for its users), offers minimal latency, and lowest cost while enhancing the overall performance.

We consider the net latency $\left(t_{i m}\right)$, net cost $\left(j_{i m}\right)$, and the corresponding utility $\left(Z_{i m}\right)$ of MVNO $m$ for each cycle or interval $i$ for updating its RF slices from WIP as metrics for performance evaluation. The net latency in each cycle or interval for updating its RF slices from WIP is expressed as

$$
t_{i m}=\sum_{w \in \hat{\mathcal{W}}} d_{w} \gamma_{w}+\eta_{n} \rho
$$

where $\gamma_{w}$ represents the latency induced by over allocation (double spending) of one RF slice, $\eta_{n}$ is the additional number of channels subleased to fulfill the user demand because of under-prediction of data rate requirements, and $\rho$ represents the system latency for renegotiation of a channel to meet the demanded rate. In case of over prediction, $\eta_{n} \rho$ is 0 thus, latency is only experienced by MVNO in case of non zero double spending. Negotiation for $\eta_{n}$ channels start after $r_{i}$ is known and when users demands is not met by current set of leased channels $\left(\hat{Q_{w}}\right)$. Similarly, the net cost $\left(j_{i m}\right)$ for a given cycle $i$ for MVNO $m$ can be defined as

$$
j_{i m}=\sum_{q_{w} \in \hat{Q}_{w}} q_{w} C_{q_{w}}
$$

Finally, the utility measure in terms of latency and cost is expressed for a given MVNO $m$ for an interval $i$ as

$$
Z_{i m}=\frac{1}{\left(1+t_{i m}\right) \times j_{i m}}
$$

where $1+t_{i m}=1$ when $t_{i m}=0$, that is, when the MVNO's 
prediction meets the user requirement and, no double spending is done against the MVNO $m$ for an interval $i$.

\section{PERFORMANCE EVALUATION}

Experimental Setup: To corroborate our analysis, we have simulated different scenarios with $\mathcal{W}=\{1,2,3,4\}$ WIPs with different number of $\mathrm{RF}$ slices that are Uniformly distributed as $U \sim[95,105]$ and double spending behavior under 4 scenarios. There are four MVNOs subleasing RF slices from WIPs to fulfil the data rate requirements of their end users for each interval by using the constraint given in (9). End users are assumed to become active to connect to the MVNO based on Poisson process and the data rate requirement of each user is assumed to be followed the Exponential distribution [13]. We assume that the inter-arrival rate of users is $\lambda=10$ and their data rate request is $\lambda^{\prime}=8 \mathrm{kbps}$ following an exponential distribution. We have assumed $\tau=3$ past blocks, and in case of less than 3 blocks available in Blockchain, total double spending (1) is calculated starting from the genesis block.

Without loss of generality, latency induced by double spending $\left(\gamma_{w}\right)$, system induced latency $(\rho)$, and block signing time by 1 validator is assumed to be $1 \mathrm{~ms}$ for simulation setup. Note that the system induced latency applies only for the under-prediction in total data rate requirement by MVNO and thus only for the corresponding negotiations. In Scenario 0, MVNOs use Blockchain to penalize double spending by using reputation system and use linear regression to predict data rate requirements. In Scenario 1, MVNOs use Blockchain to completely avoid double spending while randomly choosing the RF channels from WIPs and use linear to predict data rate requirements. In Scenario 2, MVNOs do not use Blockchain but use only linear regression for predicting data rate requirements. MVNOs in this case, randomly choose WIP RF channels for negotiation. In Scenario 3, MVNOs randomly choose RF channels from WIPs and predict the user requirement based on mean of observed data rate requirement.

For evaluating the performance, WIPs $w \in \mathcal{W}$ in interval $i$ are assumed to follow one of the following double spending patterns with no more than $L=10$ counts of total double spending : 1) increasing double spending as $d_{w i}=$ $\lfloor L \sin (i / N)\rfloor ; 2)$ decreasing double spending as $d_{w i}=\lfloor L \cos (i / \Lambda$ 3 ) constant double spending as $d_{w i}=1$; and 4) no double spending as $d_{w i}=0$, where $\mathrm{N}$ is the period. While MVNOs in scenario 1 are not affected by double spending, the approach introduces higher delay for MVNOs as allocations are done only after all validators confirm the transactions by signing the block.

Numerical Results and Discussion: First, we plotted a variation of double spending of WIPs vs. the interval or cycle for updating RF slices for MVNOs, as shown in Fig. 3 where WIP Type 1 and Type 2 have increasing and decreasing double spending respectively, and WIP Type 3 and Type 4 have one and zero double spending respectively. The scenario was chosen to assess MVNO performance across widest range of WIP behaviors. Next, Fig. 4 shows the variation of corresponding normalized reputation score for $N=30$ update intervals where we observed that reputation increases exponentially until it gets to one (max value) for WIP which is

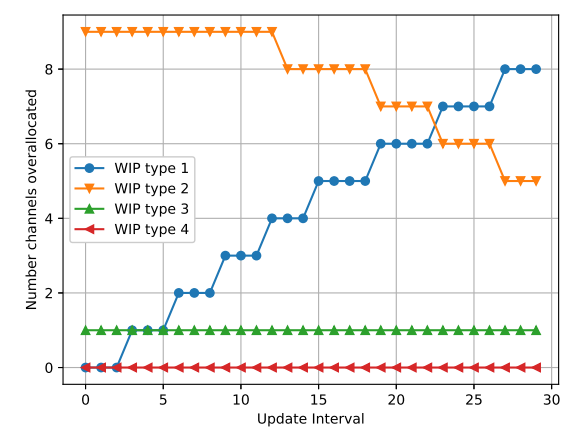

Figure 3: Double spending of WIPs versus the interval/cycle for updating RF slices for MVNOs.

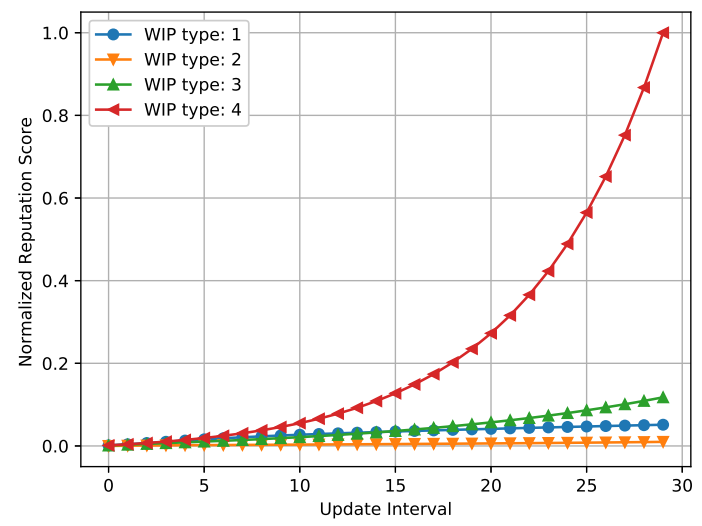

Figure 4: WIPs' normalized reputation scores for different instances.

not double spending.

Then, we plotted the variation of total cost $\left(j_{i m}\right)$ incurred for MVNOs vs. the update interval (or instances), as shown in Fig. 5. We observed that only MVNOs in Scenario 0 consistently bear the least cost in leasing the RF channels from the WIPs because of the use of Blockchain using $\mathrm{Al}$ gorithm 3 and linear regression while leasing RF slices from WIPs. Similarly, in Fig. 6, we plotted the expected latency versus the update interval, and have observed that, in Scenario 0, MVNOs resulted in the lowest delay since $(N)\rfloor$ MVNOs used both reputation based Blockchain technology

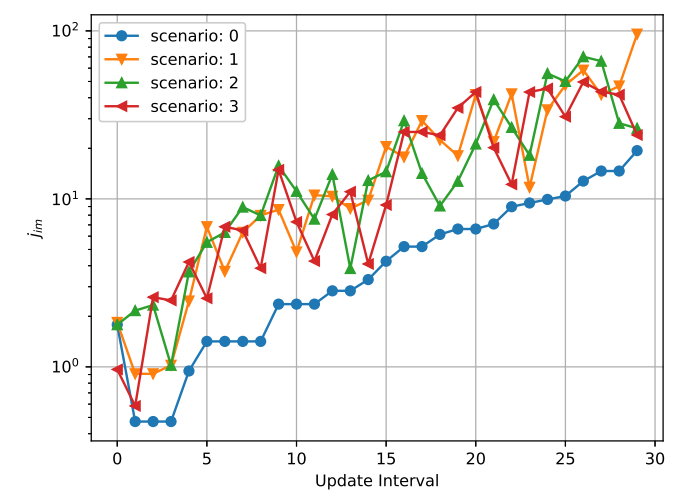

Figure 5: Expected cost incurred by different MVNO types based on the WIP chosen for leasing RF slices. 


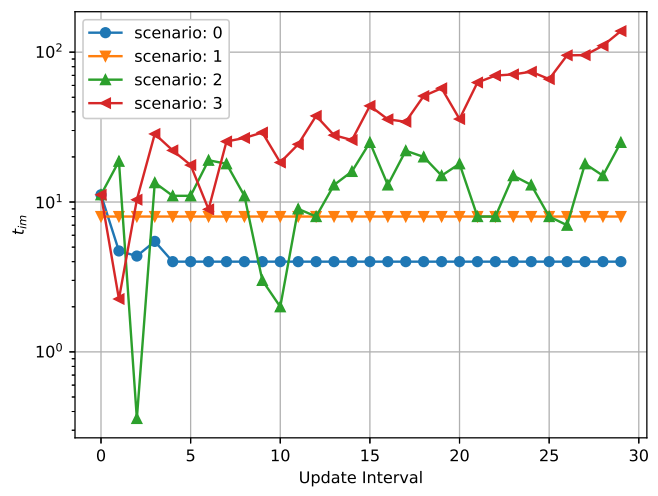

Figure 6: Expected latency for different MVNOs due to system latency (caused by misprediction) and double spending attack.

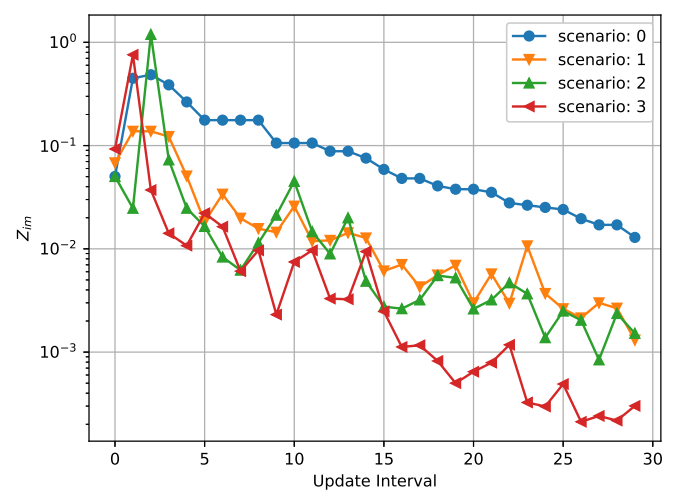

Figure 7: Expected utility vs. the interval.

and linear regression while subleasing RF slices from WIPs. In Scenario 1, MVNOs experienced a higher latency than that of Scenario 0 since each allocation is only completed after validators' confirmation is obtained, as shown in Fig. 6. Similarly, the Scenario 3 with subleasing RF slices for MVNOs randomly resulted in highest latency, as in Fig. 6.

Finally, we plotted the expected utility (12) versus the update interval, as shown in Fig. 7, for considered four scenarios. Scenario 0 and Scenario 3, respectively, resulted in the highest and lowest utilities for given setup. We can clearly observe that when the MVNO uses Blockchain based reputation scores and linear regression as in Scenario 0, the MVNOs experience relatively higher expected utility for all interval compared to any other scenarios. In Scenario 1, MVNOs use Blockchain to avoid double spending of RF slices which needs more time to process by participating validators (WIPs and MVNOs). Furthermore, MVNOs in scenario 1 have no means to optimize for price. Collectively, these two factors result in lower utility than for MVNOs in Scenario 0 (where Blockchain based reputation system is used to penalize the double spending WIPs). In the simulation, the total data rate requirement increased in every update interval, the total cost and total latency increased and thus the utility $\left(Z_{i m}\right)$ decreased with increasing update interval.

\section{CONCLUSION}

In this paper, we have proposed the joint Blockchain based reputation system to minimize double spending and linear regression based machine learning to reduce system induced delays in contract (re)negotiations for selecting RF slices of WIPs for MVNOs for wireless virtualization. Numerical results have shown that the proposed approach outperforms other approaches in terms of expected latency as well as expected utility of MVNOs for wireless virtualization.

\section{REFERENCES}

[1] D. B. Rawat, M. Song, and S. Shetty, Dynamic Spectrum Access for Wireless Networks. Springer, 2015.

[2] C. Liang and F. R. Yu, "Wireless network virtualization: A survey, some research issues and challenges," IEEE Communications Surveys $\&$ Tutorials, vol. 17, no. 1, pp. 358-380, 2015.

[3] S. Haykin, "Cognitive radio: Brain-empowered wireless communications," IEEE JSAC, vol. 23, no. 2, pp. 201-220, 2005.

[4] D. Zhang et al., "A contract-based resource allocation mechanism in wireless virtualized network," in IEEE INFOCOM 2018 Workshops, April 2018, pp. 474-479.

[5] A. Aijaz, "Hap - SliceR: A Radio Resource Slicing Framework for 5G Networks With Haptic Communications," IEEE Systems Journal, vol. 12, no. 3, pp. 2285-2296, Sept 2018.

[6] J. Z. Peter Toth, Marcel Volosin, "Agent-Based dynamic distribution of the frequency spectrum based on MASCEM implementation," in Proc. of MAC-MIME 2016, Dec 2016, pp. 199-206.

[7] D. B. Rawat and A. Alshaikhi, "Leveraging Distributed Blockchain-based Scheme for Wireless Network Virtualization with Security and QoS Constraints," in 2018 International Conference on Computing, Networking and Communications (ICNC), March 2018, pp. 332-336.

[8] Z. Lu, Q. Wang, G. Qu, and Z. Liu, "BARS: A Blockchain-Based Anonymous Reputation System for Trust Management in VANETs," in 2018 17th IEEE TrustCom/BigDataSE, Aug 2018, pp. 98-103.

[9] D. B. Rawat, A. Alshaikhi, A. Alshammari, C. Bajracharya, and M. Song, "Payoff optimization through wireless network virtualization for iot applications: A three layer game approach," IEEE Internet of Things Journal, pp. 1-1, 2019.

[10] S. Nakamoto, "Bitcoin: A peer-to-peer electronic cash system, 2008," URL (accessed on Aug. 2017): http://www.bitcoin.org/bitcoin.pdf, 2008.

[11] S. Marsland, Machine learning: an algorithmic perspective. CRC Press, 2011.

[12] F. Farahnakian, P. Liljeberg, and J. Plosila, "LiRCUP: Linear Regression Based CPU Usage Prediction Algorithm for Live Migration of Virtual Machines in Data Centers," in 39th Euromicro Conf. on Software Engineering and Advanced Applications 2019, Sept 2013, pp. 357-364.

[13] E. Nan, X. Chu, W. Guo, and J. Zhang, "User data traffic analysis for $3 \mathrm{G}$ cellular networks," in IEEE ChinaCOMM 2013, Aug 2013, pp. 468-472. 